\title{
On Lower and Upper Bounds of the Difference Between the Arithmetic and the Geometric Mean
}

\author{
By S. H. Tung
}

\begin{abstract}
Lower and upper bounds of the difference between the arithmetic and the geometric mean of $n$ quantities are given here in terms of $n$, the smallest value $a$ and the largest value $A$ of given $n$ quantities. Also, an upper bound for the difference, independent of $n$, is given in terms of $a$ and $A$. All the bounds obtained are sharp.
\end{abstract}

1. Introduction. Let $a_{1}, \ldots, a_{n}$ be $n$ quantities such that $0<a \equiv a_{1} \leqslant a_{2} \leqslant$ $\cdots \leqslant a_{n} \equiv A$. Let $A_{n}$ be their arithmetic mean, $G_{n}$ their geometric mean. Trivial lower and upper bounds of the difference $A_{n}-G_{n}$ are 0 and $A-a$ respectively. A nice upper bound has been obtained in [2]. Here we shall prove the following inequalities:

$$
n^{-1}(\sqrt{A}-\sqrt{a})^{2} \leqslant A_{n}-G_{n} \leqslant c a+(1-c) A-a^{c} A^{1-c}
$$

where

$$
c=\frac{\log [(A /(A-a)) \log A / a]}{\log A / a} .
$$

The inequalities give lower and upper bounds of the difference $A_{n}-G_{n}$ in terms of the smallest value $a$ and the largest value $A$ of the given $n$ quantities. Instead of a discrete method, a continuous and analytic approach is used to obtain the inequalities.

2. Lower Bounds. We consider the lower bound of $n$ quantities $0<a \equiv a_{1} \leqslant$ $\cdots \leqslant a_{k-1} \leqslant a_{k+1} \leqslant \cdots \leqslant a_{n} \equiv A$ with $a_{k} \equiv x, 1<k<n$, to be a variable in the interval $[a, A]$. Let the arithmetic and the geometric means of the fixed $n-1$ quantities be $A_{n-1}$ and $G_{n-1}$, respectively. Then

$$
A_{n}-G_{n}=n^{-1}\left\{(n-1) A_{n-1}+x\right\}-\left\{G_{n-1}^{n-1} x\right\}^{1 / n} \equiv D_{n}(x) \text {. }
$$

Since $D_{n}^{\prime}(x)=0$ at $x=G_{n-1}$, the lower bound of $D_{n}(x)$ for $x$ in the interval $[a, A]$ is

$$
D_{n}\left(G_{n-1}\right)=((n-1) / n)\left(A_{n-1}-G_{n-1}\right) \text {. }
$$

This result can also be found in [1, p. 12], but the method used here seems to be simpler and more straightforward. By repeating this process, we have

$$
\begin{aligned}
A_{n}-G_{n} & \geqslant \frac{n-1}{n}\left(A_{n-1}-G_{n-1}\right) \geqslant \frac{n-1}{n} \frac{n-2}{n-1}\left(A_{n-2}-G_{n-2}\right) \geqslant \cdots \\
& \geqslant \frac{2}{n}\left(A_{2}-G_{2}\right)=\frac{2}{n}\left(\frac{a+A}{2}-\sqrt{a A}\right)=\frac{1}{n}(\sqrt{A}-\sqrt{a})^{2} .
\end{aligned}
$$

Received April 18, 1974.

AMS (MOS) subject classifications (1970). Primary 26A86.

Key words and phrases. Arithmetic and geometric mean, inequality, lower and upper bounds. 
Equality holds only if $a_{2}=\cdots=a_{n-1}=\sqrt{a_{1} a_{n}}=\sqrt{a A}$.

3. Upper Bounds. Now we investigate the upper bound of $A_{n}-G_{n}$. The maximum of $D_{n}(x)$ on $[a, A]$ is attained at the endpoint $a$ or $A$. Thus, the maximum of $A_{n}-G_{n}$ of $n$ quantities is attained when $a \equiv a_{1}=\cdots=a_{k} \leqslant a_{k+1}=\cdots=a_{n} \equiv A$ for some $k, 1<k<n$, with the form

$$
\frac{k_{c}+(n-k) A}{n}-\left\{a^{k} A^{n-k}\right\}^{1 / n}=a\left[\frac{k+(n-k) A / a}{n}-\{A / a\}^{(n-k) / n}\right] .
$$

For the sake of simplicity, let $a=1$ and consider the function

$$
D(x)=\frac{x+(n-x) A}{n}-A^{(n-x) / n} .
$$

Through straight calculation, we have $D^{\prime}(x)=0$ for $x=c n$, where

$$
c=\frac{\log [(A /(A-1)) \log A]}{\log A} .
$$

Thus, the upper bound for $A_{n}-G_{n}$ is $D(c n)=c+(1-c) A-A^{1-c} \equiv U$ which is independent of $n$. By repeated application of L'Hospital's rule, we have

$$
\lim _{A \rightarrow 1} c=\frac{1}{2} \text { and } \lim _{A \rightarrow \infty} c=0 .
$$

The upper bound is attained only at its limiting case $A \rightarrow 1$ or $n \rightarrow \infty$. But this is the best possible bound independent of the positive integer $n \geqslant 2$. For a fixed $n$, the sharp upper bound of $A_{n}-G_{n}$ is attained by $D\left(k_{n}\right)$ with $k_{n}=[c n]$ or [cn] +1 , where [cn] denotes the largest integer not greater than $c n$. Therefore, we have lower and upper bounds of $A_{n}-G_{n}$, both dependent and independent of $n$, in terms of $a=1$ and $A$ as follows:

$$
0 \leqslant n^{-1}(\sqrt{A}-1)^{2} \leqslant A_{n}-G_{n} \leqslant D\left(k_{n}\right) \leqslant c+(1-c) A-A^{1-c} .
$$

Some numerical data are shown below.

\begin{tabular}{clccc}
\multicolumn{5}{c}{ TABLE $(a=1)$} \\
$A$ & \multicolumn{1}{c}{$c$} & $k_{10}$ & $D\left(k_{10}\right)$ & $U$ \\
1 & 0.5 & & 0 & 0 \\
1.001 & 0.499925 & 5 & $1.25 \times 10^{-7}$ & $1.25 \times 10^{-7}$ \\
1.1 & 0.496029 & 5 & $1.191152 \times 10^{-3}$ & $1.191227 \times 10^{-3}$ \\
2 & 0.471234 & 5 & 0.085786 & 0.086071 \\
$e$ & 0.458675 & 5 & 0.210420 & 0.211867 \\
5 & 0.434331 & 4 & 0.773472 & 0.777337 \\
10 & 0.407973 & 4 & 2.418928 & 2.419591 \\
100 & 0.333805 & 3 & 45.18114 & 45.45570 \\
$10^{5}$ & 0.212238 & 2 & $7.00002 \times 10^{4}$ & $7.00906 \times 10^{4}$ \\
$10^{10}$ & 0.136222 & 1 & $8.00000 \times 10^{9}$ & $8.20349 \times 10^{9}$
\end{tabular}


Department of Mathematics and Statistics

Miami University

Oxford, Ohio 45056

1. E. F. BECKENBACH \& R. E. BELLMAN, Inequalities, 2nd rev. ed., Ergebnisse der Mathematik und ihrer Grenzgebiete, N. F., Band 30, Springer-Verlag, New York, 1965. MR 33 \#236.

2. C. LOEWNER \& H. B. MANN, "On the difference between the geometric and the arithmetic mean of $n$ quantities," Advances in Math., v. 5, 1971, pp. 472-473. MR 43 \#4982. 